\title{
Determinación del poder corneal con un tomógrafo corneal luego de cirugía refractiva con láser excimer
} Corneal Power Determination with Corneal Tomography after
Refractive Surgery with Excimer Laser Determinação da potência corneana com uma topografia de
córnea após cirurgia refrativa com excimer láser

Luis Carlos Jaramillo, MD., Esp.*

Virgilio Galvis, MD., Esp., PhD.**

Alejandro Tello, MD., Esp., PhD.***

Paul Anthony Camacho, MD., MSc. ****

Ángela Castillo, MD., Esp.*****

Luis A. Pareja, MD.*****

\section{Resumen}

Introducción. La determinación del poder corneal, después de la cirugía refractiva con láser excimer, es difícil. Diferentes alternativas conel uso de tomógrafos corneales sehanutilizado para esta medición. Objetivo. Evaluar diversos métodos de determinación del poder corneal en pacientes operados de cirugía fotorrefractiva, incluyendo pruebas diagnósticas con medidas cuantitativas. Metodología. Estudio retrospectivo de pacientes operados de cirugía fotorrefractiva que tuvieran resultados refractivos y tomografía corneal post-operatoria al menos diez semanas luego de cirugía. Resultados. En los ojos miopes se encontraron diferencias significativas en el valor determinado por la queratometría derivada de la historia clínica al compararla con la queratometría manual promedio postoperatoria, la queratometría simulada y el Mean Pupil

*Centro oftalmológico Virgilio Galvis, Universidad Autónoma de Bucaramanga, Floridablanca, Santander, Colombia.

** Médico, especialista en Oftalmología, subespecialista en Segmento Anterior, doctorado en investigación en Cirugía y especialidades médicoquirúrgicas. Centro oftalmológico Virgilio Galvis, Universidad Autónoma de Bucaramanga, Fundación oftalmológica de Santander, Floridablanca, Santander, Colombia.

*** Médico, especialista en Oftalmología, subespecialista en Facoemulsificación y Segmento Anterior, subespecialista en Cirugía refractiva, doctorado en investigación en Cirugía y especialidades médico-quirúrgicas. Centro oftalmológico Virgilio Galvis, Universidad Autónoma de Bucaramanga, Fundación Oftalmológica de Santander, Floridablanca, Santander, Colombia.

**** Médico, magíster en Epidemiología, Universidad Autónoma de Bucaramanga, Fundación oftalmológica de Santander, Floridablanca, Santander, Colombia.

***** Centro Oftalmológico Virgilio Galvis, Floridablanca, Santander, Colombia

Correspondencia: Alejandro Tello. Fundación Oftalmológica de Santander, Centro Oftalmológico Virgilio Galvis, Floridablanca, Santander, Colombia. E-mail: alejandrotello@gmail.com. 
Power del tomógrafo Sirius ${ }^{\circledR}$. Por otra parte, al promediar la queratometría manual promedio postoperatoria con el Mean Pupil Power postoperatorio (valor denominado $\mathrm{K}_{\text {pop promedio } 1}$ ) y compararla con la queratometría derivada por el método de la historia clínica, no se observaron en los pacientes miopes diferencias estadísticamente significativas. Asimismo, al promediar la queratometría simulada postoperatoria del equipo Sirius ${ }^{\circledR}$ con el Mean Pupil Power postoperatorio (valor denominado $\mathrm{K}_{\text {pop promedio 2 }}$ ) y compararla con la queratometría derivada por el método de la historia clínica tampoco se observaron en los pacientes miopes diferencias estadísticamente significativas. Por otro lado, en los ojos hipermétropes y con astigmatismo mixto, los promedios de los errores del Mean Pupil Power postoperatorio, con respecto a la queratometría derivada por el método de la historia clínica, no fueron significativamente diferentes de los errores al comparar la $\mathrm{K}_{\text {pop promedio } 1} \mathrm{y}$ la $\mathrm{K}_{\text {pop promedio } 2}$ con la queratometría derivada por el método de la historia clínica. Conclusiones. En ojos miopes las determinaciones del poder corneal postoperatorio con las $\mathrm{K}_{\text {pop promedio } 1} \mathrm{y}$ $\mathrm{K}_{\text {pop promedio 2 }}$ se aproximan más a la queratometría derivada por el método de la historia clínica que a las mediciones de la queratometría manual promedio postoperatoria, la queratometría simulada postoperatoria o el Mean Pupil Power del equipo Sirius ${ }^{\circledR}$. [Jaramillo LC, Galvis V, Tello A, Camacho PA, Castillo A, Pareja LA. Determinación del poder corneal con un tomógrafo corneal luego de cirugía refractiva con láser excimer. MedUNAB. 2018;21(1):16-30. doi:1029375/01237047.2397].

Palabras clave: Topografía de la Córnea; Córnea; Queratectomía Fotorrefractiva; Láseres de Excímeros; Procedimientos Quirúrgicos Refractivos; Errores de Refracción.

\section{Abstract}

Introduction. The determination of corneal power, after excimer laser refractive surgery, is difficult. Different alternatives with the use of corneal tomographs have been used for this measurement. Objective. To evaluate various methods of determination of corneal power in patients undergoing photorefractive surgery, including diagnostic tests with quantitative measurements. Methodology. This is a retrospective observational study. We included patients undergoing photorefractive surgery with refractive results and postoperative corneal tomography taken at least ten weeks after surgery. Results. In the myopic eyes significant differences were found in the value determined by the keratometry derived from the clinical history when compared with the average postoperative manual keratometry, the simulated keratometry and the Mean Pupil Power of the Sirius ${ }^{\circledR}$ tomograph. On the other hand, when averaging the average postoperative manual keratometry with the postoperative Mean Pupil Power (value called $\mathrm{K}_{\text {pop average } 1}$ ) and comparing it with the keratometry derived by the clinical history method, no statistically significant differences were observed in the myopic patients. Likewise, when averaging the simulated postoperative keratometry of the Sirius ${ }^{\circledR}$ equipment with the postoperative Mean Pupil Power (value called " $K_{\text {pop average 2 }}$ ") and comparing it with the keratometry derived by the method of the clinical history, no statistically significant differences were observed in the myopic patients. On the other hand, in hyperopic eyes and with mixed astigmatism, mean errors of the postoperative Mean Pupil Power, with respect to the keratometry derived by the clinical history method, were not significantly different from the errors when comparing the " $\mathrm{K}_{\text {pop average } 1}$ "

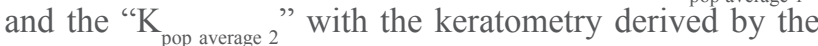
clinical history method. Conclusions. In myopic eyes the postoperative corneal power determinations with

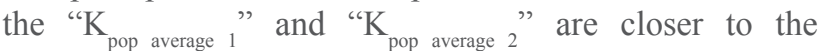
keratometry derived by the clinical history method than to the measurements of the average postoperative manual keratometry, the postoperative simulated keratometry or the Mean Pupil Power of the Sirius ${ }^{\circledR}$ equipment. [Jaramillo LC, Galvis V., Tello A., Camacho PA, Castillo A., Pareja $L A$. Corneal power determination with corneal tomography after refractive surgery with excimer laser. MedUNAB. 2018;21(1):16-30. doi:1029375/01237047.2397].

Keywords: Corneal Topography; Cornea; Photorefractive Keratectomy; Excimer Lasers; Refractive Surgical Procedures; Refractive Errors.

\section{Resumo}

Introdução. A determinação da potência corneana, após a cirurgia refrativa com excimer láser, é difícil. Foram usadas diferentes alternativas com o uso de tomógrafos de córnea para esta medição. Objetivo. Avaliar vários métodos de determinação da potência corneana em pacientes submetidos à cirurgia fotorrefrativa, incluindo testes diagnósticos com medidas quantitativas. Métodos. Este é um estudo observacional retrospectivo. Foram incluídos pacientes submetidos à cirurgia fotorrefrativa com resultados refrativos e topografía corneana pós-operatória pelo menos um mês após a cirurgia. Resultados. Nos olhos míopes foram encontradas diferenças significativas no valor determinado pela ceratectomia derivada da história clínica, quando comparada com a média da ceratectomia manual pós-operátoria, a ceratectomia simulada e o Mean Pupil Power do tomógrafo Sirius ${ }^{\circledR}$. Por outro lado, ao calcularmos a média da ceratectomia manual pós-operatória com o Mean Pupil Power pós-operatório (valor denominado $\mathrm{K}_{\text {pop média } 1}$ ) e compará-la com a ceratectomia pelo método da história 
clínica, não foram observadas diferenças estatisticamente significantes nos pacientes miopes. Da mesma forma, ao calcular a média da ceratectomia pós-operatória simulada do equipamento Sirius ${ }^{\circledR}$ com o Mean Pupil Power pós-

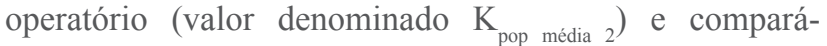
la com a ceratectomia derivada do método de história clínica, não foram observadas diferenças estatisticamente significantes nos pacientes miopes. Por outro lado, em olhos hipermetrópicos e com astigmatismo mixto, as médias de erros do Mean Pupil Power pós-operatório, em relação à ceratectomia derivada pelo método da história clínica, não foram significativamente diferentes dos erros na comparação do $\mathrm{K}_{\text {pop média } 1}$ y la $\mathrm{K}_{\text {pop média } 2}$ com a ceratectomia derivada pelo método da história clínica. Conclusões. Nos olhos míopes, as determinações de potência corneana pósoperatória com $\mathrm{K}_{\text {pop média } 1}$ e $\mathrm{K}_{\text {pop média } 2}$ estão mais próximas da ceratectomia derivada pelo método da história clínica do que das medidas de ceratectomia manual média pós-operatória, da ceratectomia simulada pós-operatória ou Mean Pupil Power do equipamento Sirius ${ }^{\circledR}$. [Jaramillo LC, Galvis $V$, Tello A, Camacho PA, Castillo A, Pareja LA. Determinação da potência corneana com uma topografia de córnea após cirurgia refrativa com excimer láser. MedUNAB. 2018;21(1):16-30. doi:1029375/01237047.2397].

Palavras-chave: Topografia da Córnea; Córnea; Ceratectomia Fotorrefrativa; Lasers de Excimer; Procedimentos Cirúrgicos Refrativos; Erros de Refração.

\section{Introducción}

Para un adecuado cálculo del poder del lente intraocular, a implantar durante la cirugía de extracción de catarata, es de vital importancia conocer el poder de la córnea del paciente. La queratometría estándar lo calcula matemáticamente mediante el uso del índice queratométrico. Este permite la determinación aproximada del poder corneal total midiendo solo el radio de curvatura de la cara anterior. Desafortunadamente, el índice queratométrico es muy impreciso en aquellos casos en que se ha producido una alteración de la superficie corneal, como, por ejemplo, luego de cirugía fotorrefractiva con láser. Desde finales de la década de 1990, diversos estudios han demostrado que este procedimiento genera sobreestimación del poder corneal total en pacientes con antecedente de corrección miópica y subestimación en quienes han recibido tratamientos hipermetrópicos $(1,2)$.

Adicionalmente, los queratómetros manuales y los topógrafos (cuando se analiza el valor de queratometría simulada $-\mathrm{SimK}$ - de estos últimos) miden los radios de curvatura con base en la reflexión de unas miras en la córnea en una zona de 3.00 $\mathrm{mm}$ de diámetro en promedio, pero no toman en consideración directamente la pequeña área central de la pupila donde se encuentra el eje visual (que es menor de, aproximadamente, $2.00 \mathrm{~mm}$ de diámetro). La medición hecha por esos sistemas del área paracentral de la córnea tiene un diámetro que puede variar entre los $2.00 \mathrm{~mm}$ y los $4.00 \mathrm{~mm}$, dependiendo tanto de las características del equipo, como de las características de curvatura de cada córnea. Esta aproximación en una córnea sin antecedentes quirúrgicos, donde cada meridiano de la córnea en el área central es casi esférico, funciona bastante bien, pues la diferencia es mínima entre una medida hecha a $3.00 \mathrm{~mm}$ y otra hecha, por ejemplo, a $1.5 \mathrm{~mm}$ de diámetro. Sin embargo, el cambio de esfericidad causada por la ablación de la superficie corneal en casos de cirugía refractiva hace que esa aproximación sea menos acertada, dadas las mayores diferencias de las curvaturas centrales y paracentrales (2-4).

Actualmente, el método introducido por Holladay en 1989, por el cual se obtiene un poder corneal derivado de la historia clínica $\left(\mathrm{K}_{\mathrm{DHC}}\right)$, es la mejor forma que tenemos para estimar el poder corneal real en un paciente operado de cirugía refractiva si, muchos años después, consulta porque presenta cataratas, de modo que se requiera tal dato para calcular el poder del lente intraocular a implantar $(4,5)$. La dificultad con este método radica en que es necesario conocer la información sobre la refracción y queratometría anteriores a la cirugíy además la de una refracción postoperatoria reciente, pero que debe ser previa a la aparición de la opacidad del cristalino (ya que la catarata nuclear puede generar miopía secundaria, alterando los cálculos). Sin embargo, como estos pacientes generalmente se operan de cirugía refractiva entre los 20 y los 40 años de edad, el lapso de tiempo hasta la aparición de la catarata usualmente es de más de 15 años (y puede ser mucho mayor dependiendo de la edad del paciente) y muy pocos pacientes regresan de manera regular a controles oftalmológicos durante este periodo de tiempo.

Por estas razones, en la mayoría de los casos, no existen datos confiables tanto preoperatorios (antes de la cirugía refractiva con láser excimer) como postoperatorios (después de la cirugía refractiva, pero antes de la aparición de la catarata). Estas circunstancias hacen que el método de la historia clínica, aunque en la teoría es muy preciso, en la práctica sea frecuentemente inaplicable o poco predecible por la 
falta de disponibilidad de datos confiables $(2,4)$.

Una posible solución para este problema consiste en estimar el poder corneal directamente en la córnea ya operada, mediante la técnica de trazado de rayos basada en la ley de Snell. A diferencia de la queratometría tradicional, la técnica de trazado de rayos no toma como base la determinación del radio de curvatura de la superficie anterior de la córnea, ni el índice queratométrico, ni la medición de zonas paracentrales. En su lugar, utiliza la medición real del radio de curvatura de las superficies corneales anterior y posterior y se basa en los verdaderos índices de refracción (aire, córnea y humor acuoso) para la estimación del poder refractivo real (6).

No existen, según los resultados de este estudio, textos de este tipo en nuestro país, y solo hay algunos publicados en el extranjero. En un estudio con este abordaje, realizado por Savini y coautores en Italia, se encontró que la diferencia entre los valores de queratometría simulada (SimK), calculados con el tomógrafo corneal Sirius ${ }^{\circledR}$ (CSO-Costruzione Strumenti Oftalmici, Florencia, Italia), antes y después de la cirugía refractiva, subestimó el cambio refractivo después de la corrección miópica y lo sobreestimó después de la corrección de la hipermetropía. Tal resultado se esperaba, dados los errores introducidos por la medición paracentral y el uso del índice queratométrico.

Por otra parte, la diferencia en la medición antes y después de la cirugía refractiva del Poder Medio Pupilar (MPP por Mean Pupil Power en inglés), calculada por el mismo equipo Sirius ${ }^{\circledR}$ con el abordaje de trazado de rayos, mostró una excelente correlación con el cambio refractivo (6). Sin embargo, aunque Savini y coautores evaluaron la diferencia de los valores de poder corneal versus el cambio refractivo, no calcularon el poder corneal postoperatorio con el método considerado entonces reglamentario (el método de la historia clínica, $\mathrm{K}_{\mathrm{DHC}}$ ), para poder compararlo con el determinado en el postoperatorio por el MPP $(4,6)$. Otros estudios sobre la determinación de los cambios corneales luego de cirugía corneal con láser excimer han sido realizados por Holladay y colaboradores en los Estados Unidos, utilizando el tomógrafo Pentacam ${ }^{\circledR}$ (Oculus, Wetzlar, Alemania) (7), por Sónego-Krone y colaboradores en Brasil, con el tomógrafo corneal Orbscan $\mathrm{II}^{\circledR}$ (Bausch \& Lomb, Orbtek Inc., Salt Lake City, Estados Unidos) (8), y por Gelender en los Estados Unidos, también con el Orbscan II (9).
El presente estudio tiene como objetivo evaluar la concordancia del MPP determinado por el equipo Sirius $^{\mathbb{R}}$ o de un valor derivado de esa medición junto con otras mediciones postoperatorias directas del poder corneal, luego de cirugía refractiva con láser excimer, con el poder calculado por el método de la historia clínica de Holladay $\left(\mathrm{K}_{\mathrm{DHC}}\right)$, el cual hasta el momento aún se considera como la "regla de oro" si se dispone de datos confiables pre- y postoperatorios $(4,5)$. Este equipo se denomina tomógrafo corneal porque, además de capturar y analizar información de la cara anterior de la córnea (por reflexión de discos de Plácido), también obtiene datos de la cara posterior (usando una hendidura de luz), a diferencia de los topógrafos corneales basados únicamente en reflexión, que solo capturan información de la cara anterior de la córnea. Ahora, la determinación directa del poder corneal real luego de cirugía refractiva sería muy útil para ser aplicada en el futuro en otros pacientes con este antecedente, pero en quienes no conozcamos la información previa al procedimiento con láser excimer, situación que como, ya se indicó, es muy frecuente.

\section{Metodología}

Este estudio es de carácter retrospectivo observacional. Analiza la correlación de diversos métodos para la determinación del poder corneal luego de cirugía refractiva, incluyendo pruebas diagnósticas con mediciones cuantitativas. Se incluyeron en el estudio todos aquellos pacientes sin antecedente de otra cirugía corneal, a quienes se les practicó cirugía fotorrefractiva con láser excimer en el Centro Oftalmológico Virgilio Galvis, que alcanzaron una agudeza visual con corrección postoperatoria mayor de 20/40 y a quienes se les realizó tomografía corneal con el tomógrafo Sirius ${ }^{\circledR}$ al menos diez semanas luego del procedimiento. Como criterios de exclusión se consideró la presentación de alguna complicación intraoperatoria o postoperatoria, o algún tipo de comorbilidad retiniana. Se analizó el equivalente esférico pre- y postoperatorio y la queratometría manual promedio pre- $\mathrm{y}$ postoperatoria (oftalmómetro OM-4, Topcon, Itabashi-Ku, Japón). Adicionalmente, se evaluaron el SimK y el MPP postoperatorios, medidos con el tomógrafo Sirius ${ }^{\circledR}$. Se compararon estos valores con el poder corneal calculado por el método de historia clínica de Holladay $\left(\mathrm{K}_{\mathrm{DHC}}\right)$, Este método consiste en determinar el cambio refractivo generado por la cirugía, ajustarlo al vértice corneal y, luego, sustraer (en el caso de ojos operados de miopía) o adicionar (en el caso de ojos hipermétropes) ese 
cambio al poder corneal preoperatorio, para obtener así el poder corneal postoperatorio $(4,5)$.

Para explicar brevemente este método utilizaremos un ejemplo. Si un paciente tenía una refracción preoperatoria, medida de la manera estándar (es decir, en el plano de las gafas, aproximadamente $12 \mathrm{~mm}$ del vértice corneal) de -5.25 Dioptrías (D), y luego de la cirugía refractiva se encuentra en emetropía (es decir, con un error de cero D), el método se aplica de la manera indicada a continuación:

Primero se toman los valores de las refracciones medidas en el plano de las gafas y se convierten en refracciones medidas en el vértice de la córnea, con una fórmula sencilla (10):

$\mathrm{Rc}=\mathrm{Rg} /[1-(\mathrm{g} * \mathrm{Rg})]$

En donde:

"Rc" es la refracción corregida al plano de la córnea, considerando la distancia al vértice corneal.

"Rg" es la refracción medida en el plano de las gafas (en el examen clínico usual).

"g" es la distancia a la cual se midió la refracción con respecto al vértice de la córnea (en metros), conocida como "distancia al vértice", que corresponde a la distancia de la córnea a la que se encontrarán los lentes colocados en unas gafas. Esta distancia varía entre 10 y $15 \mathrm{~mm}$ (es decir, entre 0.01 y $0.015 \mathrm{~m}$ ), dependiendo del instrumento que se emplee para colocar los lentes de prueba frente al ojo del paciente, aunque usualmente es de $12 \mathrm{~mm}(0.012 \mathrm{~m})$.

En el ejemplo, las refracciones pre- y postoperatorias corregidas al vértice de la córnea, aplicando la fórmula mencionada, resultan:

Refracción preoperatoria corregida al vértice corneal: $-4.94 \mathrm{D}$

Refracción postoperatoria corregida al vértice corneal: $0 \mathrm{D}$

Para el siguiente paso, en el método derivado de la historia clínica, se calcula entonces la diferencia en refracción pre- y postoperatoria en el plano de la córnea. Al valor de refracción preoperatoria se le resta el de la refracción postoperatoria.
En nuestro ejemplo: $(-4.94 \mathrm{D})-(0)=-4.94 \mathrm{D}$.

Este es el valor en el cual, efectivamente, se disminuyó el poder de la córnea del paciente. En seguida, se toma el poder corneal medido por queratometría en el preoperatorio, que, supongamos, era de $45.00 \mathrm{D}$; a este valor se le suma el cambio refractivo y, de esa manera, se calcula el poder corneal postoperatorio. En el caso de miopía, se le sumará un valor negativo; es decir, se disminuirá el poder corneal, que es lo que efectivamente hace la cirugía.

En nuestro ejemplo:

$\mathrm{K}_{\mathrm{DHC}}=45.00 \mathrm{D}+(-4.94 \mathrm{D})=40.06 \mathrm{D}$.

Este es el poder corneal real postoperatorio de ese ojo. Este valor se podría utilizar para ser introducido en una fórmula de cálculo del poder de un lente intraocular cuando el paciente requiera cirugía de catarata (siempre y cuando la refracción postoperatoria se haya realizado poco tiempo antes de la aparición de la catarata, condición que, como se mencionó anteriormente, se cumple en muy pocos casos, porque los pacientes no regresan de manera regular a controles anuales).

En el grupo de pacientes del presente estudio, dado que habían sido operados unos meses antes, todos tenían la información completa para aplicar el método de la historia clínica con el fin de determinar el poder corneal real postoperatorio. Por supuesto en ninguno de ellos se requería este dato para calcular un lente intraocular, pues no tenían catarata, sino que se hizo para usarlo como referencia en la evaluación de la precisión del método directo, o de valores derivados de ese método, con el tomógrafo Sirius ${ }^{\circledR}$. Con este propósito se obtuvieron los promedios de dos mediciones postoperatorias diferentes (ambas incluyendo como uno de los términos el MPP): $\mathrm{Km}_{\mathrm{pop}}$ promediada con el MPP (valor denominado en el presente estudio $\mathrm{K}_{\text {pop promedio } 1}$ ) y $\operatorname{SimK} \mathrm{K}_{\text {pop }}$ promediada con el MPP (valor denominado en el presente estudio $\mathrm{K}_{\text {pop promedio 2 }}$ ). Estos dos valores se compararon con la $\mathrm{K}_{\mathrm{DHC}}$

El análisis descriptivo se realizó según la distribución de frecuencias. Las variables cualitativas se presentaron en frecuencias absolutas y relativas. En las variables cuantitativas se evaluó la normalidad, mediante la prueba de Shapiro Wilk, gráficamente $\mathrm{y}$ estadísticamente. Las diferencias entre las medidas del poder corneal postoperatorio directo 
$\left(\mathrm{Km}_{\mathrm{pop}}\right.$ SimK $\left.\mathrm{pop}_{\mathrm{pop}}\right)$ y la derivada por el método de la historia clínica $\left(\mathrm{K}_{\mathrm{DHC}}\right)$ se evaluaron con la prueba de $\mathrm{t}$ de Student pareada. La relación entre la $\mathrm{K}_{\text {pop promedio } 1} \mathrm{y}$ la $\mathrm{K}_{\text {pop promedio } 2}$ con la $\mathrm{K}_{\mathrm{DHC}}$ se evaluó con el coeficiente de correlación de Pearson y se calculó ésta mediante una regresión lineal robusta. El acuerdo entre la $\mathrm{K}_{\mathrm{DHC}}$ con cada uno de estos promedios (la $\mathrm{K}_{\text {pop promedio } 1} \mathrm{y}$ la $\mathrm{K}_{\text {pop promedio } 2}$ ) se evaluó mediante el gráfico de límites de Bland-Altman.

Es importante considerar que dos métodos que miden una misma variable biológica deben ser concordantes e intercambiables. Se espera que la diferencia media entre estos no sea diferente de cero y que el $95 \%$ de las diferencias estén dentro de los percentiles 2.5 y 97.5; además, que estas diferencias no sean clínicamente importantes. Los límites, de acuerdo con esto, pueden tener diferencias entre las mediciones, sin que lleguen a tener significancia clínica e, idealmente, deberían definirse de antemano para ayudar en la interpretación de la comparación de los diferentes métodos (1114). Para este estudio, los límites de las diferencias máximas aceptables serían de $1.00 \mathrm{D}$ para la medida del poder corneal postoperatorio. El análisis se realizó en el software STATA VE 11.2 y el nivel de significancia fue del 5\% (12,13).

La investigación se adhirió a las recomendaciones de la Declaración de Helsinki de Octubre del 2008 $\mathrm{y}$ todas sus actualizaciones y fue aprobada por el Comité de Ética en Investigaciones de la Fundación Oftalmológica de Santander (CEI - FOSCAL). Debido a la naturaleza observacional y retrospectiva del estudio, no se consideró necesario un consentimiento informado por parte de los pacientes incluidos, cuya identidad se mantuvo siempre en anonimato.

\section{Resultados}

Se analizaron 92 ojos (46 ojos derechos y 46 izquierdos) de 50 pacientes. Todos los pacientes habían sido sometidos a cirugía fotorrefractiva con láser excimer entre diciembre de 2012 y agosto de 2015. Las características demográficas de los pacientes se encuentran en la Tabla 1. En el 84.78\% (78 ojos) se realizó queratomileusis asistida por láser excimer (LASIK, por Laser assisted in Situ Keratomileusis) y, en el $15.22 \%$ (14 ojos), queratectomía fotorrefractiva transepitelial con láser excimer (Trans-PRK, por Transepithelial Photorefractive Keratectomy).

El seguimiento promedio fue de 4.31 meses y el rango, de 2.8 a 9.3 meses. Los miopes se dividieron en dos grupos: miopes bajos, con equivalente esférico menos negativo que - 5.00 D (39 ojos), y miopes altos, con equivalente esférico igual o más negativo que - 5.00 D (14 ojos). Se analizaron por separado también los ojos con defectos hipermetrópicos (29 ojos) y con astigmatismo mixto (10 ojos). Tanto en los ojos miopes como en los ojos hipermétropes, la queratometría promedio manual postoperatoria $\left(\mathrm{Km}_{\mathrm{pop}}\right)$ subestimó los cambios generados por la cirugía refractiva (Tabla 2).

Al comparar la queratometría derivada por el método de la historia clínica $\left(\mathrm{K}_{\mathrm{DHC}}\right)$ con la queratometría promedio manual postoperatoria $\left(\mathrm{Km}_{\mathrm{pop}}\right)$, se observó en los miopes altos una diferencia de algo más de media $\mathrm{D}$, siendo la $\mathrm{K}_{\mathrm{DHC}}$ menor que la $\mathrm{Km}_{\text {pop }}$ (37.96 +/- $0.98 \mathrm{D}$ versus $38.59+/-1.10 \mathrm{D}, \mathrm{p}=0.0002$ ). En el grupo de pacientes miopes bajos, esta comparación mostró una diferencia de algo menos de media $\mathrm{D}$ en la misma dirección $\left(\mathrm{K}_{\mathrm{DHC}}=40.94+/-1.69 \mathrm{D}\right.$ versus $\left.\mathrm{Km}_{\mathrm{pop}}=41.43+/-1.56 \mathrm{D}, \mathrm{p}<0.001\right)$. En hipermétropes, la diferencia fue de algo más de media $\mathrm{D}$, pero mostrando mayor curvatura la $\mathrm{K}_{\mathrm{DHC}}$ que la $\mathrm{Km}_{\text {pop }}$ $(46.57+/-2.13 \mathrm{D}$ versus $46.01+/-1.98 \mathrm{D}, \mathrm{p}=0.001)$. En pacientes con astigmatismo mixto, la diferencia fue de solo un sexto de dioptría aproximadamente $(43.41+/-1.85 \mathrm{D}$ versus $43.58+/-2.48, \mathrm{p}=0.687)$ (Tabla 3).

Al comparar la $\mathrm{K}_{\mathrm{DHC}}$ con la queratometría postoperatoria determinada por el SimK del tomógrafo Sirius $^{\circledR}\left(\operatorname{SimK}_{\text {pop }}\right)$, se observó en los miopes altos una diferencia de algo más de tres cuartos de $\mathrm{D}$, siendo la $\mathrm{K}_{\mathrm{DHC}}$ menor que la $\operatorname{SimK}_{\text {pop }}(37.96+/-0.98 \mathrm{D}$ versus $38.73+/-0.78 \mathrm{D}, \mathrm{p}=0.03$ ). En el grupo de pacientes miopes bajos, esta comparación mostró una diferencia de algo menos de media $\mathrm{D}$ en la misma dirección $\left(\mathrm{K}_{\mathrm{DHC}}\right.$ $=40.94+/-1.69 \mathrm{D}$ versus $\operatorname{SimK}_{\mathrm{pop}}=41.38+/-1.61 \mathrm{D}$, $\mathrm{p}=0.25)$. En hipermétropes, la diferencia fue de algo menos de media $\mathrm{D}$, pero mostró mayor curvatura la $\mathrm{K}_{\mathrm{DHC}}$ que la $\operatorname{SimK}_{\text {pop }}(46.57+/-2.13 \mathrm{D}$ versus 46.10 $+/-2.16 \mathrm{D}, \mathrm{p}=0.41)$. En pacientes con astigmatismo mixto, la diferencia fue cercana a cero $(43.60+/-1.86$ $\mathrm{D}$ versus $43.58+/-2.48, \mathrm{p}=0.98$ ) (Tabla 3).

Al comparar la $\mathrm{K}_{\mathrm{DHC}}$ con el MPP postoperatorio en los miopes altos, se observó una subestimación del poder corneal por parte del MPP $(37.45+/-0.83 \mathrm{D}$ versus $37.96+/-0.98 \mathrm{D}, \mathrm{p}=0.0002$ ), es decir una tendencia contraria a la observada al comparar la $\mathrm{K}_{\mathrm{DHC}}$ con la $\mathrm{Km}_{\text {pop }}$ o con la $\mathrm{SimK}_{\mathrm{pop}}$. En el grupo de pacientes miopes bajos, se encontró una diferencia algo mayor de 
Tabla 1. Características demográficas (50 individuos / 92 ojos)

\begin{tabular}{ll}
\hline Característica & \\
\hline Edad & $31.4 \pm 10.26$ \\
[Promedio \pm Desv. Estándar (rango)] & $(17-55)$ años \\
\hline Sexo: Femenino, $\mathbf{n}(\%)$ & $36(72 \%)$ \\
\hline Defecto refractivo original & \\
\hline Defecto miópico & 30 individuos / 53 ojos (57.6\%) \\
\hline Defecto hipermetrópico & 16 individuos* / 29 ojos (31.5\%) \\
\hline Astigmatismo mixto & 7 individuos* / 10 ojos (10.9\%) \\
\hline
\end{tabular}

* Dos pacientes presentaban un defecto hipermetrópico en un ojo y astigmatismo mixto en el otro; una paciente, un defecto miópico en un ojo y astigmatismo mixto en el otro. Por esta razón, el número de individuos, al sumar los tres grupos, resulta en 53, pero es de 50. La unidad de análisis de cada grupo fueron los resultados de cada ojo.

Tabla 2. Cambios inducidos por la cirugía en el equivalente esférico y la queratometría promedio (medida con queratómetro manual)

\begin{tabular}{|c|c|c|c|c|c|c|}
\hline $\begin{array}{l}\text { Medidas pre-y } \\
\text { postoperatorias } \\
\text { Defecto } \\
\text { Refractivo }\end{array}$ & $\begin{array}{l}\text { EE* preope- } \\
\text { ratorio (pla- } \\
\text { no corneal). } \\
\text { Dioptrías }\end{array}$ & $\begin{array}{l}\text { EE* postope- } \\
\text { ratorio (pla- } \\
\text { no corneal). } \\
\text { Dioptrías }\end{array}$ & $\begin{array}{l}\text { Diferencia } \\
\text { EE* (plano } \\
\text { corneal). } \\
\text { Dioptrías }\end{array}$ & $\begin{array}{l}\text { Queratome- } \\
\text { tría prome- } \\
\text { dio preope- } \\
\text { ratoria. } \\
\text { Dioptrías }\end{array}$ & $\begin{array}{l}\text { Queratome- } \\
\text { tría prome- } \\
\text { dio posto- } \\
\text { peratoria. } \\
\text { Dioptrías }\end{array}$ & $\begin{array}{l}\text { Diferencia } \\
\text { en quera- } \\
\text { tometría } \\
\text { promedio. } \\
\text { Dioptrías }\end{array}$ \\
\hline $\begin{array}{l}\text { Miopes bajos } \\
\text { ( } n=39 \text { ojos) }\end{array}$ & $-2.93 \pm 0.92$ & $0.07 \pm 0.42$ & $-3.00 \pm 1.07 * \star$ & $43.94 \pm 1.21$ & $41.43 \pm 1.56$ & $-2.51 \pm 1.11^{\star \star}$ \\
\hline $\begin{array}{l}\text { Miopes altos } \\
\text { ( } n=14 \text { ojos) }\end{array}$ & $-6.24 \pm 0.69$ & $-0.09 \pm 0.6$ & $-6.14 \pm 0.72^{\star \star}$ & $44.11 \pm 1.07$ & $38.59 \pm 1.09$ & $-5.52 \pm 0.96^{\star *}$ \\
\hline $\begin{array}{l}\text { Hipermétropes } \\
\text { ( } n=29 \text { ojos) }\end{array}$ & $2.64 \pm 1.66$ & $-0.54 \pm 1.13$ & $3.18 \pm 1.73^{\star \star}$ & $43.39 \pm 1.44$ & $46.01 \pm 1.98$ & $2.62 \pm 1.63^{\star \star}$ \\
\hline $\begin{array}{l}\text { Astígmatismo } \\
\text { mixto ( } n=10 \text { ojos) }\end{array}$ & $0.01 \pm 1.09$ & $-0.16 \pm 0.50$ & $0.17 \pm 1.54$ & $43.41 \pm 1.43$ & $43.41 \pm 1.85$ & $0.00 \pm 1.75$ \\
\hline
\end{tabular}

*EE: Equivalente esférico.

$* *$ Prueba t de Student pareada, $\mathrm{p}<0.001$ 
un tercio de $\mathrm{D}$ también con subestimación por parte del $\operatorname{MPP}(40.56+/-1.75 \mathrm{D}$ versus $40.94+/-1.69 \mathrm{D}, \mathrm{p}=0.001)$. En el grupo de pacientes hipermétropes, se encontró una diferencia algo menor de un tercio de dioptría, con subestimación del poder por parte del MPP (46.27 +/$2.33 \mathrm{D}$ versus $46.57+/-2.13 \mathrm{D}, \mathrm{p}=0.033$ ). En el grupo de astigmatismo mixto, la diferencia fue de un poco menos de una quinta parte de una $\mathrm{D}(43.58+/-2.48 \mathrm{D}$ versus $43.39+/-2.18 \mathrm{D}, \mathrm{p}=0.465$ ) (Tabla 3).

En la Tabla 4 se indican los valores de p para las comparaciones de las diferencias entre la $\mathrm{K}_{\mathrm{DHC}} \mathrm{y}$ la $\mathrm{Km}_{\text {pop }}$, con las diferencias de la $\mathrm{K}_{\mathrm{DHC}}$ y el MMP postoperatorio; así como las diferencias de la $\mathrm{K}_{\mathrm{DHC}}$ versus la $\mathrm{SimK}_{\text {pop }}$ para los cuatro grupos de ojos (miopes altos, miopes bajos, hipermétropes y ojos con astigmatismo mixto). Se encontró que, tanto en hipermétropes, como en ojos con astigmatismo mixto, no hubo diferencias significativas entre las diversas magnitudes de las diferencias entre los otros métodos, con respecto al de la historia clínica, ni al comparar sus valores aritméticos (teniendo en cuenta los signos) ni al comparar sus valores absolutos.

Ahora, por otra parte, en los ojos miopes sí se encontraron diferencias significativas entre los valores cuando se analizaron teniendo en cuenta los signos, pues, mientras que, con respecto al valor determinado por la $\mathrm{K}_{\mathrm{DHC}}$, tanto la $\mathrm{Km}_{\text {pop }}$ como la $\mathrm{SimK}_{\mathrm{pop}}$ sobrevaloraron el poder corneal postoperatorio en los ojos miopes, el MPP lo subvaloró. Es decir, el error se presentó en la dirección contraria. Sin embargo, al analizar los valores absolutos de ese error, con respecto a la $\mathrm{K}_{\mathrm{DHC}}$, no se identificó una diferencia estadísticamente significativa entre la $\mathrm{Km}_{\text {pop}}$, la $\operatorname{SimK}_{\text {pop }}$ y el MPP. Esto nos sugirió que la magnitud del error era similar, pero en dirección contraria (Tabla 4).

Teniendo en cuenta ese detalle, consideramos posible que, al promediar el MPP con alguna de las otras dos mediciones posoperatorias, en ojos miopes, los

Tabla 3. Diferencia entre la queratometría derivada por el método de la historia clínica $\left(\mathrm{K}_{\mathrm{DHC}}\right)$ y la queratometría manual postoperatoria $\left(\mathrm{Km}_{\mathrm{pop}}\right)$, la queratometría simulada del equipo $\operatorname{Sirius}^{\circledR}\left(\mathrm{SimK}_{\mathrm{pop}}\right)$ y el Mean Pupil Power postoperatorio (MPP), según defecto refractivo*

\begin{tabular}{|c|c|c|c|c|c|c|}
\hline & $\mathrm{K}_{\mathrm{DHC}}$ vs. $\mathrm{Km}_{\mathrm{pop}}$ & & $\mathrm{K}_{\mathrm{DHC}}$ vs. SimK $\mathrm{pop}_{\mathrm{p}}$ & & $\mathrm{K}_{\mathrm{DHC}}$ VS. MPP & \\
\hline & Promedio \pm DS & $\mathrm{p}^{* *}$ & Promedio \pm DS & $p^{* *}$ & Promedio \pm DS & $p^{\star *}$ \\
\hline $\begin{array}{l}\text { Grupo completo de } \\
\text { Miopes } \\
\text { ( } n=53 \text { ojos) }\end{array}$ & $-0.53 \pm 0.54$ & $<0.001$ & $-0.53 \pm 0.48$ & 0.1619 & $+0.42 \pm 0.49$ & $<0.001$ \\
\hline $\begin{array}{l}\text { Miopes altos }>-5.00 \mathrm{D} \\
(\mathrm{n}=14 \text { ojos })\end{array}$ & $-0.63 \pm 0.46$ & 0.0002 & $-0.77 \pm 0.42$ & 0.03 & $+0.52 \pm 0.37$ & 0.0002 \\
\hline $\begin{array}{l}\text { Miopes bajos } \leq 5.00 \mathrm{D} \\
\text { ( } n=39 \text { ojos })\end{array}$ & $-0.49 \pm 0.56$ & $<0.001$ & $-0.44 \pm 0.47$ & 0.25 & $+0.38 \pm 0.52$ & 0.001 \\
\hline $\begin{array}{l}\text { Hipermétropes } \\
\text { ( } n=29 \text { ojos) }\end{array}$ & $+0.56 \pm 0.73$ & 0.001 & $+0.47 \pm 0.66$ & 0.41 & $+0.30 \pm 0.73$ & 0.033 \\
\hline $\begin{array}{l}\text { Astigmatismo mixto } \\
\text { ( } n=10 \text { ojos) }\end{array}$ & $+0.17 \pm 1.28$ & 0.687 & $-0.02 \pm 0.91$ & 0.98 & $+0.19 \pm 0.81$ & 0.465 \\
\hline
\end{tabular}

*Valores en dioptrías

**Test $\mathrm{t}$ de Student pareada, $\mathrm{p}<0.005$

DS=Desviación estándar 
errores tuvieran tendencia a cancelarse. Por ello, como nuevas posibilidades para determinar el poder corneal postoperatorio se calculó el promedio de los datos del MPP postoperatorio y la $\mathrm{Km}_{\mathrm{pop}}$, por un lado (denominándose este promedio $\mathrm{K}_{\text {pop pomedio }}$ ), y del MPP postoperatorio y la $\mathrm{SimK}_{\mathrm{pop}}$, por otro lado (llamándolo $\mathrm{K}_{\text {pop promedio } 2}$ ). En el grupo de miopes altos la media de la $\mathrm{K}_{\text {pop promedio } 1}$ fue de $38.02+/-0.95 \mathrm{D}$, que no fue estadísticamente diferente del valor de $37.96+/-0.98$ $\mathrm{D}$ calculado por $\mathrm{K}_{\mathrm{DHC}}(\mathrm{p}=0.871)$. La media de la $\mathrm{K}_{\text {pop promedio } 2}$ fue de $38.09+/-0.80 \mathrm{D}$, que tampoco fue estadísticamente diferente del calculado por el método de la historia clínica $(\mathrm{p}=0.704)$. En el grupo de miopes bajos, la media de la $\mathrm{K}_{\text {pop promedio } 1}$ fue de 41.00 +/- 1.64 $\mathrm{D}$, lo que no fue estadísticamente diferente del valor de $40.94+/-1.69 \mathrm{D}$ calculado por la $\mathrm{K}_{\mathrm{DHC}}(\mathrm{p}=0.874)$. La media de la $\mathrm{K}_{\text {pop promedio } 2}$ fue de $40.97+/-1.68 \mathrm{D}$, lo que tampoco fue estadísticamente diferente del cálculo por $\mathrm{K}_{\mathrm{DHC}}(\mathrm{p}=0.938)$. Las magnitudes de las diferencias de cada uno de estos dos valores $\left(\mathrm{K}_{\text {pop promedio } 1} \mathrm{y}\right.$ $\mathrm{K}_{\text {pop promedio } 2}$ ), con respecto a los poderes calculados por la $\mathrm{K}_{\mathrm{DHC}}$, se muestran en la Tabla 5. Las diferencias promedio de la $\mathrm{K}_{\text {pop promedio } 1}$ y la $\mathrm{K}_{\text {pop promedio } 2}$ comparadas con la $\mathrm{K}_{\mathrm{DHC}}$ versus la diferencia del MPP con respecto a la $\mathrm{K}_{\mathrm{DHC}}$ se muestran en la Tabla 6. Se obtuvieron fórmulas de regresión para calcular la $\mathrm{K}_{\mathrm{DHC}}$ a partir de

Tabla 4. Comparación de las diferencias entre el poder derivado por el método de la historia clínica $\left(\mathrm{K}_{\mathrm{DHC}}\right)$ y el poder corneal de la queratometría manual, la SimK y el Mean Pupil Power (MPP) postoperatorios, según defecto refractivo

\begin{tabular}{|c|c|c|c|c|c|c|}
\hline \multirow{2}{*}{$\begin{array}{l}\text { Comparación de las } \\
\text { diferencias con res- } \\
\text { pecto al } \mathrm{K}_{\mathrm{DHC}}\end{array}$} & \multicolumn{3}{|c|}{$\begin{array}{l}\text { Valores artiméticos de las diferencias* } \\
\text { (valores de } p)^{\star *}\end{array}$} & \multicolumn{3}{|c|}{$\begin{array}{l}\text { Valores absolutos de las diferencias* } \\
\text { (valores de } \mathrm{p})^{\star \star}\end{array}$} \\
\hline & $\begin{array}{l}\mathrm{K}_{\mathrm{DHC}} / \mathrm{Km}_{\mathrm{pOp}} \\
\mathrm{vs.}_{\mathrm{DHC}} / \mathrm{K}^{\mathrm{M}} \\
\mathrm{MPP}\end{array}$ & 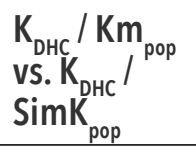 & $\begin{array}{l}\mathrm{K}_{\mathrm{DHC}} / \mathrm{MPP} \\
\text { vs. } \mathrm{K}_{\mathrm{DHC}} / \\
\operatorname{SimK}_{\text {pop }} \\
\end{array}$ & $\begin{array}{l}\mathrm{K}_{\mathrm{DHC}} / \mathrm{Km}_{\mathrm{pop}} \\
\text { vs. } \mathrm{K}_{\mathrm{DHC}} /{ }^{\mathrm{MPP}} \\
\end{array}$ & 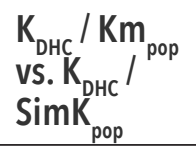 & $\begin{array}{l}\mathrm{K}_{\mathrm{DHC}} / \mathrm{MPP} \\
\text { vs. } \mathrm{K}_{\mathrm{DHC}} / \\
\text { SimK }_{\text {pop }}\end{array}$ \\
\hline $\begin{array}{l}\text { Grupo completo de } \\
\text { Miopes } \\
\text { ( } n=53 \text { ojos) }\end{array}$ & $\begin{array}{l}-0.53 \text { vs. } \\
+0.42 \\
(<0.0001)\end{array}$ & $\begin{array}{c}-0.53 \text { vs. }-0.53 \\
(1.00)\end{array}$ & $\begin{array}{c}+0.42 \\
\text { vs. }-0.53 \\
(<0.0001)\end{array}$ & $\begin{array}{l}0.53 \text { vs. } 0.42 \\
(0.2746)\end{array}$ & $\begin{array}{l}0.53 \text { vs. } 0.53 \\
\quad(1.00)\end{array}$ & $\begin{array}{l}0.42 \text { vs. } 0.53 \\
\quad(0.246)\end{array}$ \\
\hline $\begin{array}{l}\text { Miopes al- } \\
\text { tos }>-5.00 \mathrm{D} \\
(\mathrm{n}=14 \text { ojos })\end{array}$ & $\begin{array}{l}-0.63 \text { vs. } \\
+0.52 \\
(<0.0001)\end{array}$ & $\begin{array}{c}-0.63 \text { vs. }-0.77 \\
(0.408)\end{array}$ & $\begin{array}{c}+0.52 \\
\text { vs. }-0.77 \\
(<0.0001)\end{array}$ & $\begin{array}{l}0.63 \text { vs. } 0.52 \\
(0.4919)\end{array}$ & $\begin{array}{l}0.63 \text { vs. } 0.77 \\
(0.408)\end{array}$ & $\begin{array}{l}0.52 \text { vs. } 0.77 \\
(0.107)\end{array}$ \\
\hline $\begin{array}{l}\text { Miopes bajos } \leq 5.00 \\
\text { D } \\
\text { ( } n=39 \text { ojos })\end{array}$ & $\begin{array}{c}-0.49 \text { vs. } \\
+0.38 \\
(<0.0001)\end{array}$ & $\begin{array}{c}-0.49 \text { vs. }-0.44 \\
(0.674)\end{array}$ & $\begin{array}{c}+0.38 \\
\text { vs. }-0.44 \\
(<0.0001)\end{array}$ & $\begin{array}{c}0.49 \text { vs. } 0.38 \\
(0.377)\end{array}$ & $\begin{array}{l}0.49 \text { vs. } 0.44 \\
(0.674)\end{array}$ & $\begin{array}{c}0.38 \text { vs. } 0.44 \\
(0.595)\end{array}$ \\
\hline $\begin{array}{l}\text { Hipermétropes } \\
\text { ( } n=29 \text { ojos })\end{array}$ & $\begin{array}{l}+0.56 \text { vs. } \\
+0.30(0.178)\end{array}$ & $\begin{array}{l}+0.56 \text { vs. } \\
+0.47(0.621)\end{array}$ & $\begin{aligned} & +0.30 \text { vs. } \\
+ & 0.47(0.356)\end{aligned}$ & $\begin{array}{l}0.56 \text { vs. } 0.30 \\
(0.178)\end{array}$ & $\begin{array}{l}0.56 \text { vs. } 0.47 \\
(0.621)\end{array}$ & $\begin{array}{l}0.30 \text { vs. } 0.47 \\
(0.356)\end{array}$ \\
\hline $\begin{array}{l}\text { Astigmatismo mixto } \\
\text { ( } n=10 \text { ojos) }\end{array}$ & $\begin{array}{l}+0.17 \text { vs. } \\
+0.19(0.967)\end{array}$ & $\begin{array}{l}+0.17 \text { vs. } \\
-0.02(0.707)\end{array}$ & $\begin{array}{l}+0.19 \text { vs. } \\
-0.02(0.747)\end{array}$ & $\begin{array}{l}0.17 \text { vs. } 0.19 \\
(0.967)\end{array}$ & $\begin{array}{l}0.17 \text { vs. } 0.02 \\
(0.707)\end{array}$ & $\begin{array}{l}0.19 \text { vs. } 0.02 \\
(0.794)\end{array}$ \\
\hline
\end{tabular}

*Valores en dioptrías

**Test $\mathrm{t}$ de Student pareada, $\mathrm{p}<0.005$

$\mathrm{K}_{\mathrm{DHC}}$ : Método de la historia clínica

$\mathrm{Km}_{\text {pop }}$ : Queratometría manual posotperatoria

MPP:Poder Pupila Medio del tomógrafo Sirius ${ }^{\circledR}$

SimK $_{\text {pop }}$ : Queratometría simulada del tomógrafo Sirius ${ }^{\circledR}$ postoperatoria 
Tabla 5. Diferencia entre la $\mathrm{K}_{\text {DHC }}$ y la $\mathrm{K}_{\text {pop promedio } 1}$, y entre la $\mathrm{K}_{\text {DHC }}$ y la $\mathrm{K}_{\text {pop promedio } 2}$

\begin{tabular}{|c|c|c|c|c|}
\hline & \multicolumn{2}{|c|}{$\mathrm{K}_{\mathrm{DHC}}$ VS $\mathrm{K}_{\text {pop promedio } 1}$} & \multicolumn{2}{|l|}{$\mathrm{K}_{\mathrm{DHC}} \mathrm{Vs} \mathrm{K}_{\text {pop promedio 2 }}$} \\
\hline & Promedio \pm DS & $p^{*}$ & Promedio \pm DS & $p^{*}$ \\
\hline $\begin{array}{l}\text { Grupo completo de Miopes } \\
\text { ( } n=53 \text { ojos) }\end{array}$ & $-0.06 \pm 0.44$ & 0.878 & $-0.06 \pm 0.46$ & 0.877 \\
\hline $\begin{array}{l}\text { Miopes altos }>-5.00 D \\
\text { ( } n=14 \text { ojos })\end{array}$ & $-0.06 \pm 0.34$ & 0.871 & $-0.13 \pm 0.38$ & 0.704 \\
\hline $\begin{array}{l}\text { Miopes bajos } \leq 5.00 \mathrm{D} \\
(\mathrm{n}=39 \text { ojos })\end{array}$ & $-0.06 \pm 0.47$ & 0.874 & $-0.03 \pm 0.48$ & 0.937 \\
\hline $\begin{array}{l}\text { Hipermétropes } \\
\text { ( } n=29 \text { ojos) }\end{array}$ & $+0.43 \pm 0.6$ & 0.444 & $+0.39 \pm 0.68$ & 0.5 \\
\hline $\begin{array}{l}\text { Astigmatismo mixto } \\
\text { ( } n=10 \text { ojos) }\end{array}$ & $+0.18 \pm 1.01$ & 0.86 & $+0.09 \pm 0.85$ & 0.93 \\
\hline
\end{tabular}

*Test t de Student pareada, $\mathrm{p}<0.005$

$\mathrm{K}_{\mathrm{DHC}}$ : Potencia corneal derivada por el método de la historia clínica

$\mathrm{K}_{\text {pop promedio } 1}=\left(\mathrm{Km}_{\text {pop }}+\mathrm{MMP}\right) / 2$

$\mathrm{K}_{\text {pop promedio 2 }}=\left(\mathrm{SimK}_{\text {pop }}+\mathrm{MMP}\right) / 2$

la $\mathrm{K}_{\text {pop promedio 1 }} \mathrm{y}$ de la $\mathrm{K}_{\text {pop pomedio2 }}$ para los cuatros grupos de ojos (miopes altos, miopes bajos, hipermétropes y ojos con astigmatismo mixto) (Figuras 1 y 2). Para los ojos miopes, se construyeron los gráficos de Bland y Altman para las determinaciones de la $\mathrm{K}_{\text {pop promedio } 1} \mathrm{y}$ la $\mathrm{K}_{\text {pop promedio } 2}$ comparadas con el poder corneal calculado por el método de la historia clínica $\left(\mathrm{K}_{\mathrm{DHC}}\right)$ (Figuras 3 y 4). Los límites de acuerdo de estas diferencias, estuvieron por debajo del límite preestablecido, como de significancia clínica (Figuras 3 y 4).

\section{Discusión}

La determinación del poder corneal con un queratómetro (o solo los datos de la superficie anterior de la córnea obtenidos con un topógrafo o tomógrafo corneal) utiliza el índice queratométrico, que permite obtener ese valor (aproximado) sin necesidad de medir la curvatura de la superficie posterior de la córnea, lo cual constituye un abordaje con resultados bastante cercanos a la realidad en córneas vírgenes. Por otra parte, en pacientes que han sido sometidos a cirugía fotorrefractiva, la relación entre las curvaturas corneal anterior y posterior se altera significativamente $y$, por ello, al aplicar el índice queratométrico se obtienen resultados erróneos.

En general, en ojos operados de miopía, los instrumentos que utilizan este índice tienden a sobreestimar el valor del poder corneal. En los casos de ojos hipermétropes, por el contrario, existe una tendencia a subestimar el valor real del poder corneal. Esto introduce un error adicional al momento de usar fórmulas biométricas para el cálculo del poder de los lentes intraoculares cuando estos pacientes se someten a cirugía de catarata $(2,3,15)$.

Una alternativa que ofrece la tecnología actual es la determinación del poder corneal sin tener que asumir valores de la curvatura corneal posterior, sino midiéndola directamente con un tomógrafo corneal y realizando el cálculo del poder corneal real con un abordaje de trazado de rayos, es decir, con una prueba diagnóstica cuantitativa. El equipo Sirius ${ }^{\circledR}$ denomina a esta alternativa el poder medio pupilar (en inglés, Mean Pupil Power o MPP). Para poder determinar la precisión de esta medida, se puede comparar con el cálculo del poder corneal basado en el método de la historia clínica, el cual se fundamenta en la 
Tabla 6. Comparación de las diferencias entre el poder derivado por el método de la historia clínica ( $\mathrm{K}_{\mathrm{DHC}}$ ) y el MPP comparado con las diferencias entre el entre el poder derivado por el método de la historia clínica $\left(\mathrm{K}_{\mathrm{DHC}}\right)$

y el poder corneal calculado por la $\mathrm{K}_{\text {pop promedio } 1}$ y por la $\mathrm{K}_{\text {pop promedio } 2^{\prime}}$, según defecto refractivo

\begin{tabular}{|c|c|c|c|c|}
\hline \multirow{2}{*}{$\begin{array}{l}\text { Comparación de las } \\
\text { diferencias con res- } \\
\text { pecto al } \mathrm{K}_{\mathrm{DHC}}\end{array}$} & \multicolumn{2}{|c|}{$\begin{array}{l}\text { Valores aritméticos de las diferencias* } \\
\text { (valores de } \mathrm{p})^{\star \star}\end{array}$} & \multicolumn{2}{|c|}{$\begin{array}{l}\text { Valores absolutos de las diferencias* } \\
\text { (valores de } \mathrm{p})^{\star \star}\end{array}$} \\
\hline & $\begin{array}{l}\mathrm{K}_{\mathrm{DHC}} / \mathrm{MMP} \text { vs } \\
\mathrm{K}_{\mathrm{DHC}} / \mathrm{K}_{\text {pop promedio } 1}\end{array}$ & $\begin{array}{l}\mathrm{K}_{\mathrm{DHC}} / \mathrm{MMP} \text { vs } \\
\mathrm{K}_{\mathrm{DHC}} / \mathrm{K}_{\text {pop promedio } 2}\end{array}$ & $\begin{array}{l}\mathrm{K}_{\mathrm{DHC}} / \mathrm{MMP} \text { vs } \\
\mathrm{K}_{\mathrm{DHC}} / \mathrm{K}_{\text {pop promedio } 1}\end{array}$ & $\begin{array}{l}\mathrm{K}_{\mathrm{DHC}} / \mathrm{MMP} \text { vs } \\
\mathrm{K}_{\mathrm{DHC}} / \mathrm{K}_{\text {pop promedio 2 }}\end{array}$ \\
\hline $\begin{array}{l}\text { Grupo completo de } \\
\text { Miopes } \\
\text { ( } n=53 \text { ojos) }\end{array}$ & $\begin{array}{c}+0.42 \text { vs }-0.06 \\
(<0.0001)\end{array}$ & $\begin{array}{c}+0.42 \text { vs }-0.06 \\
(<0.0001)\end{array}$ & $\begin{array}{l}0.42 \text { vs } 0.06 \\
(<0.0001)\end{array}$ & $\begin{array}{l}0.42 \text { vs } 0.06 \\
(<0.0001)\end{array}$ \\
\hline $\begin{array}{l}\text { Miopes al- } \\
\text { tos }>-5.00 D \\
(n=14 \text { ojos })\end{array}$ & $\begin{array}{c}+0.52 \text { vs }-0.06 \\
(0.0002)\end{array}$ & $\begin{array}{c}+0.52 \text { vs }-0.13 \\
(0.001)\end{array}$ & 0.52 vs $0.06(0.002)$ & 0.52 vs $0.13(0.01)$ \\
\hline $\begin{array}{l}\text { Miopes bajos } \leq \\
5.00 \mathrm{D} \\
\text { ( } n=39 \text { ojos })\end{array}$ & $\begin{array}{l}+0.38 \text { vs }-0.06 \\
(0.0002)\end{array}$ & $\begin{array}{c}+0.38 \text { vs }-0.03 \\
(0.006)\end{array}$ & 0.38 vs $0.06(0.006)$ & 0.38 vs $0.03(0.003)$ \\
\hline $\begin{array}{l}\text { Hipermétropes } \\
\text { ( } n=29 \text { ojos) }\end{array}$ & $\begin{array}{c}+0.30 \text { vs }+0.43 \\
(0.462)\end{array}$ & $\begin{array}{c}+0.30 \text { vs }+0.39 \\
(0.629)\end{array}$ & 0.30 vs $0.43(0.462)$ & 0.30 vs $0.39(0.629)$ \\
\hline $\begin{array}{l}\text { Astigmatismo mixto } \\
\text { ( } n=10 \text { ojos) }\end{array}$ & $\begin{array}{c}+0.19 \text { vs }+0.18 \\
(0.98)\end{array}$ & $\begin{array}{c}+0.19 \text { vs }+0.09 \\
(0.791)\end{array}$ & 0.19 vs $0.18(0.98)$ & 0.19 vs 0.09 (0.791) \\
\hline
\end{tabular}

* Valores en dioptrías.

**Test $\mathrm{t}$ de Student pareada, $\mathrm{p}<0.005$

$\mathrm{K}_{\mathrm{DHC}}$ : Método de la historia clínica

MPP:Poder Pupilar Medio del tomógrafo Sirius ${ }^{\circledR}$

$\mathrm{K}_{\text {pop promedio } 1}=\left(\mathrm{Km}_{\text {pop }}+\mathrm{MMP}\right) / 2$

$\mathrm{K}_{\text {pop promedio 2 }}=\left(\mathrm{SimK}_{\text {pop }}+\mathrm{MMP}\right) / 2$

sustracción del cambio refractivo medido en el plano corneal, de acuerdo con lo descrito por Holladay, ya explicado en Metodología $(1,2,4,5,15)$.

Savini y colaboradores, en su estudio publicado en 2014, incluyeron 72 ojos a los que se les practicó cirugía fotorrefractiva y encontraron que la diferencia ente el MPP preoperatorio y postoperatorio tuvo una excelente correlación con el cambio refractivo inducido por la cirugía. Aunque esos autores compararon las diferencias entre los valores pre- y postoperatorios de la queratometría simulada y del MPP, por separado, no determinaron el poder corneal postoperatorio por el método de Holladay (6). Nuestro abordaje fue un poco diferente: evaluamos la correlación entre el
MPP postoperatorio y la queratometría postoperatoria derivada por el método de historia clínica, sin necesidad de tener el dato del MPP preoperatorio. Encontramos una correlación de este valor con el obtenido por el método de la queratometría derivada según la historia clínica $\left(\mathrm{K}_{\mathrm{DHC}}\right)$.

Inicialmente, obtuvimos fórmulas de regresión para el cálculo de la queratometría derivada por el método de la historia clínica a partir del MPP. Sin embargo, tanto la queratometría manual como la Sim K postoperatorias con el equipo Sirius ${ }^{\circledR}$ en los ojos miopes sobrevaloraron el poder corneal, con respecto al poder derivado por el método de la historia clínica; el MPP postoperatorio con el Sirius ${ }^{\circledR}$ lo subvaloró. 

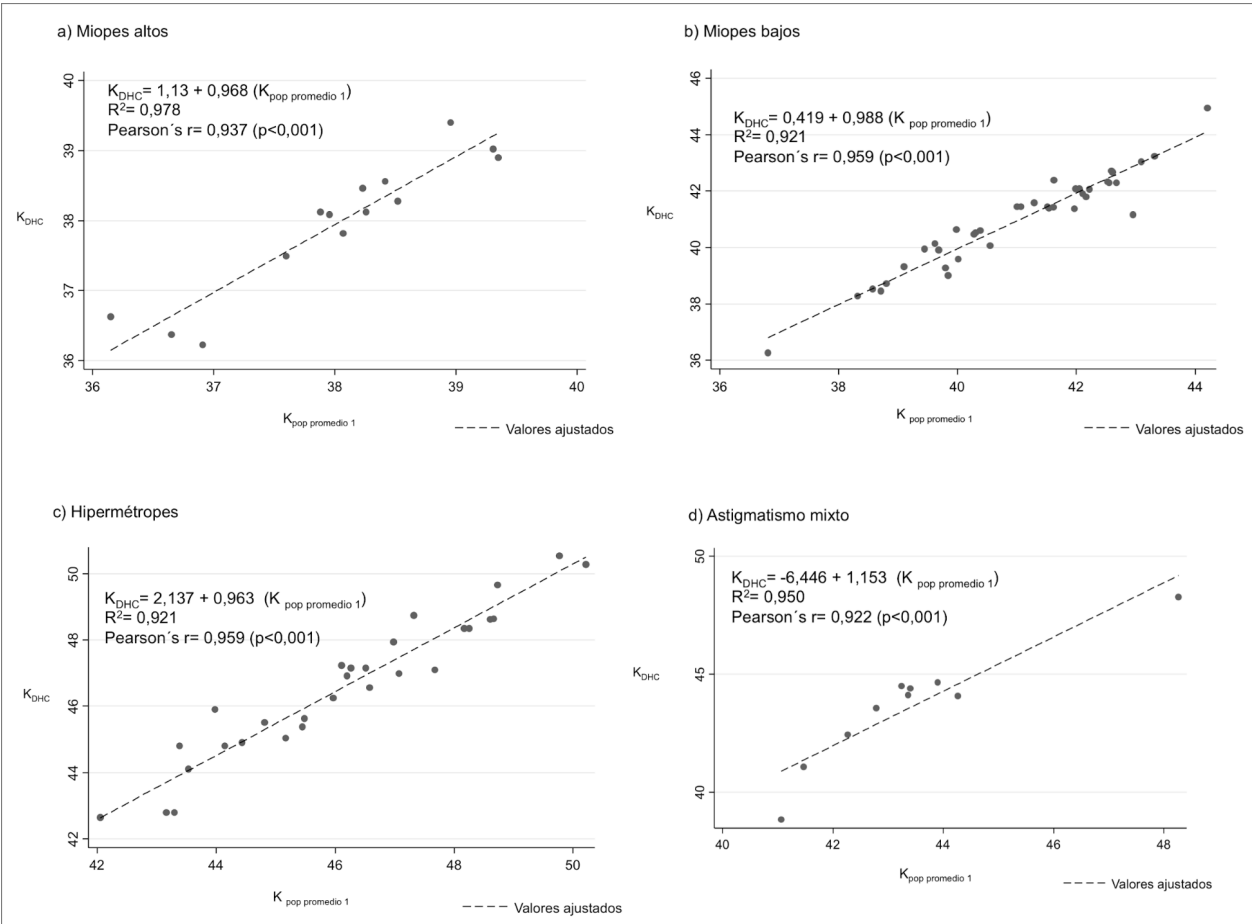

Figura 1. Correlación de $\mathrm{K}_{\text {pop promedio } 1} \mathrm{y}_{\mathrm{DHC}}$ en pacientes operados de cirugía refractiva. a) Miopes altos; b) Miopes bajos; c) Hipermétropes; d) Astigmatismo mixto
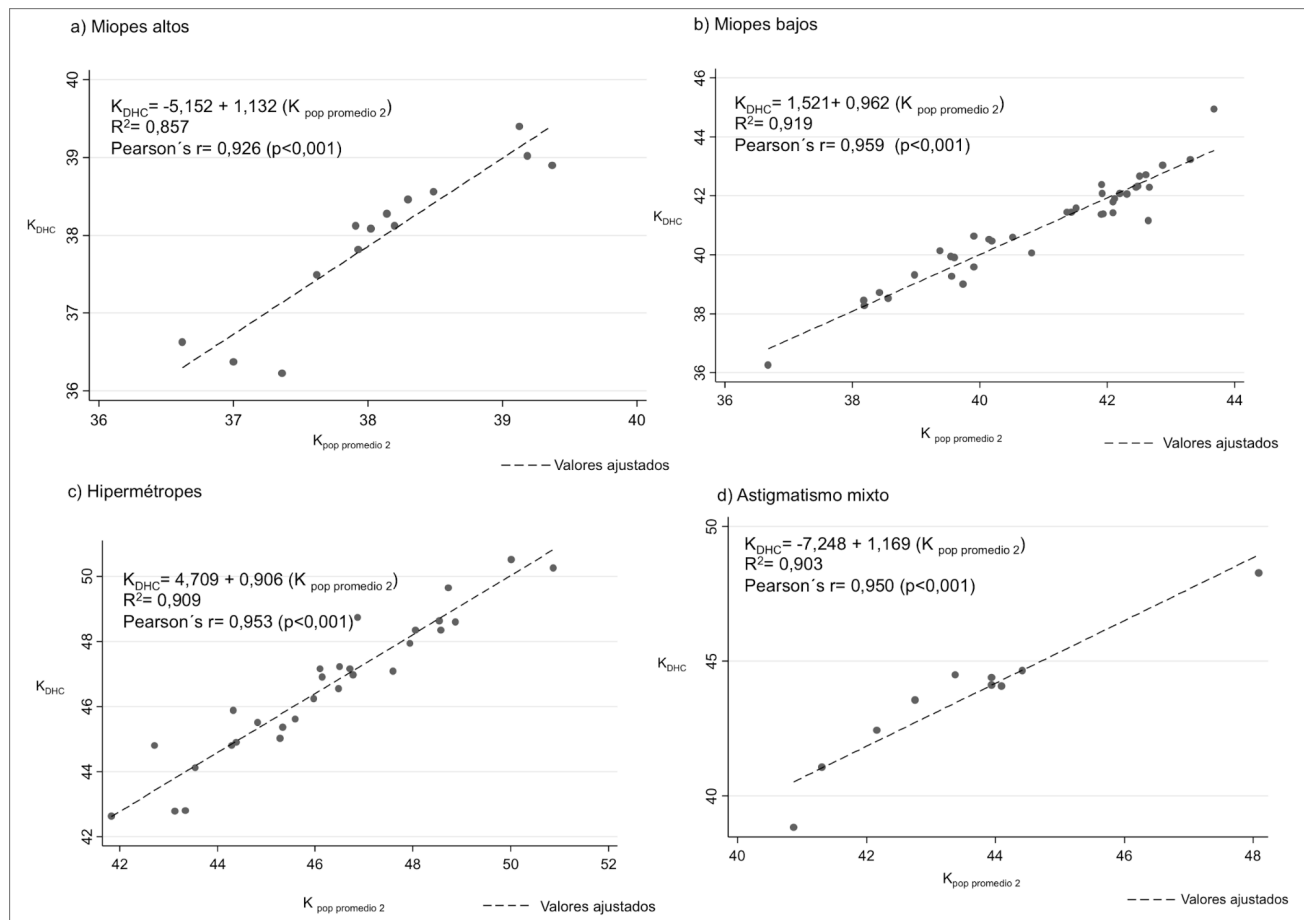

Figura 2. Correlación de $\mathrm{K}_{\text {pop promedio } 2} \mathrm{y} \mathrm{K}_{\mathrm{DHC}}$ en pacientes operados de cirugía refractiva. a) Miopes altos; b) Miopes bajos; c) Hipermétropes; d) Astigmatismo mixto 

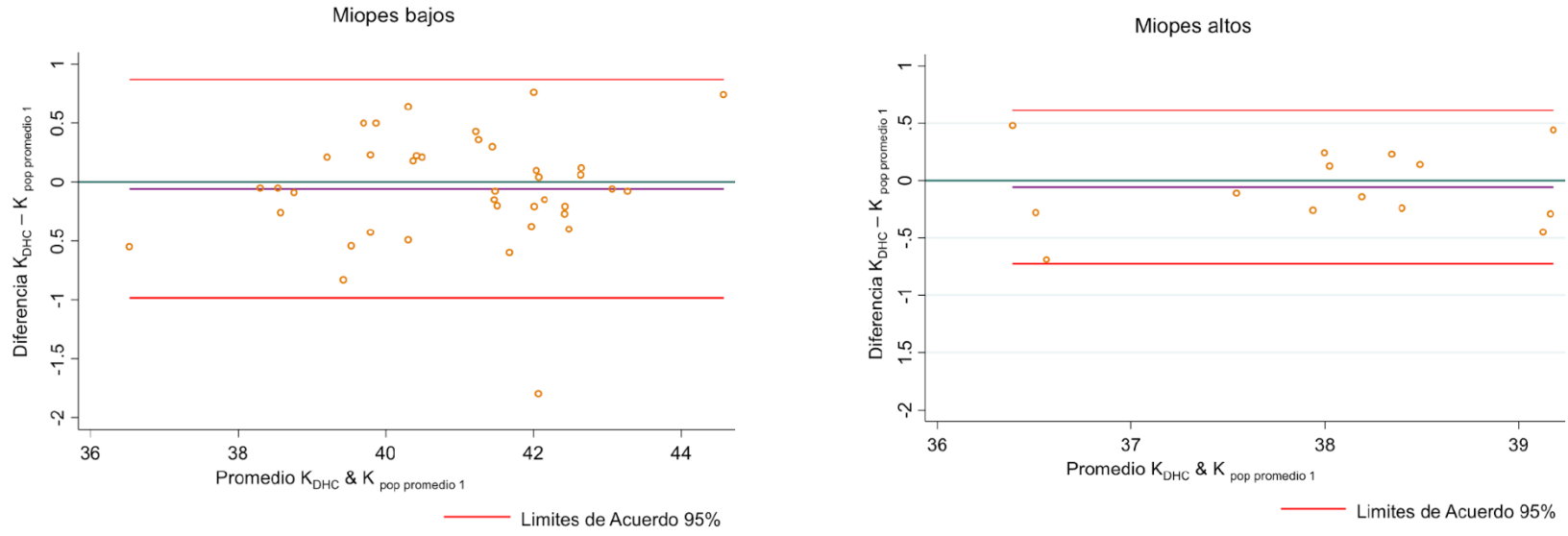

Figura 3. Gráficas de Bland y Altman para las determinaciones de la $\mathrm{K}_{\text {pop promedio } 1}$ comparadas con el poder corneal calculado por el método de la historia clínica $\left(\mathrm{K}_{\mathrm{DHC}}\right)$ en ojos miopes
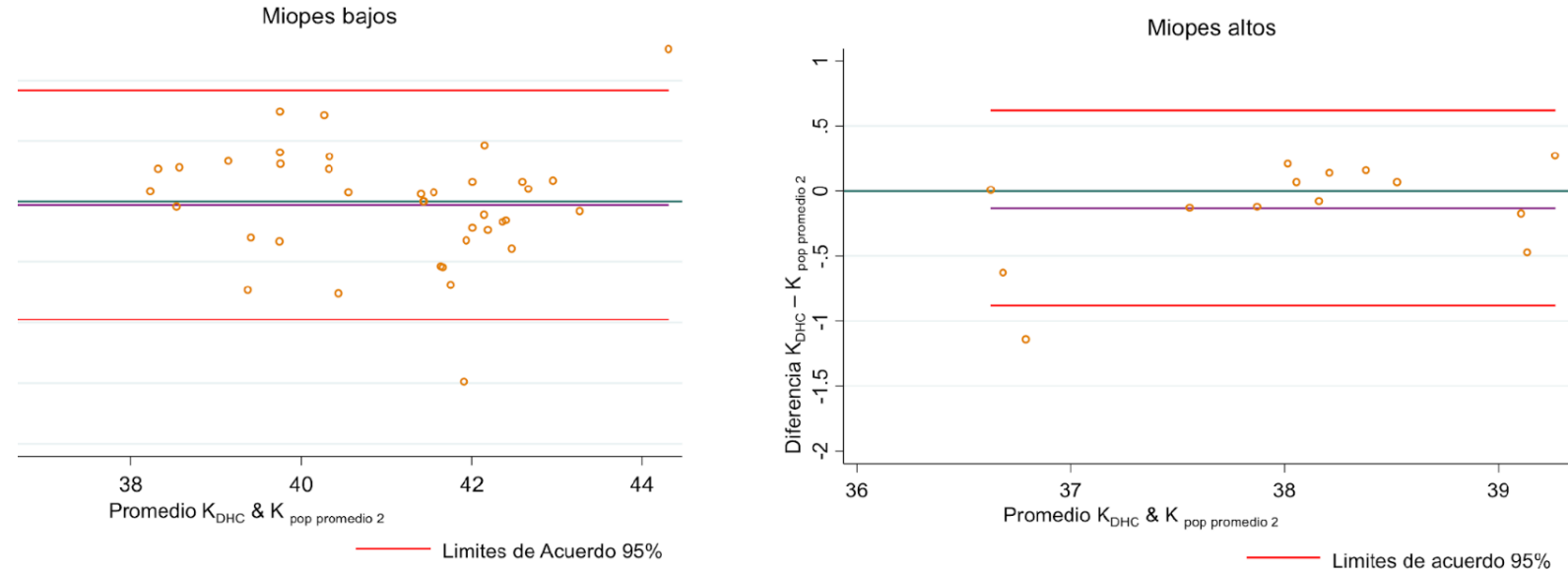

Figura 4. Gráficas de Bland y Altman para las determinaciones de la $\mathrm{K}_{\text {pop promedio } 2}$ comparadas con el poder corneal calculado por el método de la historia clínica $\left(\mathrm{K}_{\mathrm{DHC}}\right)$ en ojos miopes

Las magnitudes del error eran similares, pero en direcciones opuestas. Ante ello, decidimos calcular un promedio de los datos de la queratometría manual posoperatoria y el MPP postoperatorio, por un lado $\left(\mathrm{K}_{\text {pop promedio } 1}\right)$, y del SimK $\mathrm{K}_{\text {pop }}$ y el MPP postoperatorio, por otro lado ( $\left.\mathrm{K}_{\text {pop promedio } 2}\right)$, y compararlos con el poder derivado por el $\mathrm{K}_{\mathrm{DHC}}$, previendo que los errores en direcciones contrarias se cancelarían. Efectivamente, los valores calculados por estos dos promedios fueron muy cercanos a los poderes corneales calculados por el método de la historia clínica $\left(\mathrm{K}_{\mathrm{DHC}}\right)$, sin mostrar diferencias estadísticamente significativas con este.
Las magnitudes de las diferencias de estos dos valores $\left(\mathrm{K}_{\text {pop promedio } 1}\right.$ y $\mathrm{K}_{\text {pop promedio } 2}$ ), con respecto a la $\mathrm{K}_{\mathrm{DHC}}$, fueron muy bajas (Tabla 5). Las diferencias promedio de estas magnitudes, comparadas con el poder corneal calculado por el método de la historia clínica $\left(\mathrm{K}_{\mathrm{DHC}}\right)$, fueron significativamente menores en los ojos miopes que las diferencias del MPP postoperatorio aislado, con respecto al método de la historia clínica $\left(\mathrm{K}_{\mathrm{DHC}}\right)$ (Tabla 6). No ocurrió así en los ojos hipermétropes ni con astigmatismo mixto. Encontramos una correlación de los valores $\mathrm{K}_{\text {pop promedio } 1} \mathrm{y} \mathrm{K}_{\text {pop promedio } 2}$ con el obtenido por el $\mathrm{K}_{\mathrm{DHC}}$ Obtuvimos fórmulas de 
regresión para el cálculo de la $\mathrm{K}_{\mathrm{DHC}}$ a partir de las $\mathrm{K}_{\text {pop promedio } 1} \mathrm{y} \mathrm{K}_{\text {pop promedio 2 }}$ (Figuras 1 y 2).

Al comparar la $\mathrm{K}_{\text {pop promedio } 1}$ y la $\mathrm{K}_{\text {pop promedio } 2}$ con la $\mathrm{K}_{\mathrm{DHC}}$, utilizando las gráficas de Bland y Altman, los límites de acuerdo para los ojos miopes estuvieron por debajo del límite preestablecido como de significancia clínica (Figuras 3 y 4 ).

En el estudio realizado por Holladay y colaboradores, publicado en 2009 (7), se utilizó el equivalent K-reading (EKR) de otro equipo de tomografía corneal (Pentacam ${ }^{\circledR}$, Oculus, Wetzlar, Alemania). Para medir el poder corneal central, se obtuvo una desviación media de $-0.06 \pm 0.56 \mathrm{D}$ en 100 pacientes sometidos a LASIK, aunque también con un rango amplio (-1.63 a + 1.34 D).

Por otra parte, Sónego-Krone y colaboradores, en un trabajo publicado en 2004, utilizaron múltiples mapas de poder corneal obtenidos por el tomógrafo corneal Orbscan II $^{\circledR}$ (Bausch \& Lomb, Orbtek Inc., Salt Lake City, Estados Unidos). Encontraron en 26 ojos que el Total-Mean Power en el área de $2 \mathrm{~mm}$ de diámetro mostraba una diferencia promedio con respecto al cambio refractivo calculado al plano corneal de 0.07 $\pm 0.62 \mathrm{D}$; sin embargo, no se indicó el rango de esta diferencia (aunque sí, que un ojo presentaba una diferencia mayor a 1.00 D). El Total-Optical Power realizó una mejor evaluación del poder corneal a 4 $\mathrm{mm}$ de diámetro central, con una diferencia media de $-0.08 \pm 0.53 \mathrm{D}$. El rango no fue indicado y dos ojos presentaron diferencias mayores a $1.00 \mathrm{D}(8)$.

En el estudio de Gelender, publicado en el 2006, se analizó 59 ojos sometidos a LASIK miópico; de ellos, se derivó el poder corneal a partir de los mapas de poder medio (Mean Power Maps) del Orbscan $\mathrm{II}^{\circledR}$ y se determinó el área de 1.5 y $2 \mathrm{~mm}$ centrales como la más precisa para realizar esta medida. Posteriormente, después de aplicar este abordaje en 17 pacientes sometidos a cirugía de catarata, obtuvo una desviación media de error refractivo postoperatorio de $+0.05 \pm$ $0.59 \mathrm{D}$ con un rango de $-0.75 \mathrm{a}+0.90 \mathrm{D}$. Esto sugiere que estas determinaciones del poder corneal en pacientes con cirugía refractiva previa, obtenidas por medio de un tomógrafo corneal, podrían ser aplicadas de forma precisa dentro de las fórmulas empleadas para realizar el cálculo del lente intraocular cuando estos pacientes requieran cirugía de catarata (9).

En nuestro estudio, al combinar dos métodos para establecer el poder corneal postoperatorio luego de cirugía refractiva [uno a partir de la medición del radio de curvatura de la cara anterior, la queratometria manual $\left(\mathrm{Km}_{\text {pop }}\right)$, y otro a partir de la medida del MPP del tomógrafo Sirius ${ }^{\circledR}$ ] en ojos con antecedente de defectos miópicos, encontramos que el valor determinado no fue estadísticamente diferente del calculado por el método de la historia clínica $\left(\mathrm{K}_{\mathrm{DHC}}\right)$ $\mathrm{y}$ obtuvo unos promedios de diferencias medias de entre -0.03 y $-0.13 \mathrm{D}$. Estos errores medios fueron significativamente menores que los encontrados al comparar el MPP con el método de la historia clínica, en ojos miopes. Adicionalmente, al emplear el método de Bland y Altman, se encontró que los límites de acuerdo de las diferencias entre estas medias y la $\mathrm{K}_{\mathrm{DHC}}$ estuvieron por debajo del nivel preestablecido como de significancia clínica (que fue de 1.00 D). Por ello, proponemos estos dos promedios $\left(\mathrm{K}_{\text {pop promedio } 1} \mathrm{y} \mathrm{K}_{\text {pop promedio } 2}\right)$ como una alternativa válida para determinar el poder corneal postoperatorio en ojos con antecedente de cirugía refractiva con láser excimer para miopía. Para confirmar la intercambiabilidad de estos valores, se requiere una validación con un número mayor de ojos y, además, en ojos con antecedente de cirugía refractiva con láser excimer, que sean sometidos a cirugía de extracción de catarata por facoemulsificación.

En ojos hipermétropes y con astigmatismo mixto, los resultados fueron menos concluyentes, ya que no se encontró una clara ventaja al utilizar el MPPpop sobre la $\mathrm{Km}_{\text {pop, }}$ o la $\mathrm{SimK}_{\text {pop }}$ con respecto a sus errores en comparación con la $\mathrm{K}_{\mathrm{DHC}}^{\text {pop }}$ (Tabla 3). Tampoco se observaron diferencias estadísticamente significativas al emplear los valores derivados compuestos $\mathrm{K}_{\text {pop promedio } 1} \mathrm{y} \mathrm{K}_{\text {pop.promedio 2 } 2}$ ni en ojos con defecto previo hipermetrópico, ni en ojos con defecto previo de astigmatismo mixto (Tabla 6).

Las limitaciones del presente estudio incluyen su carácter retrospectivo, por lo cual debimos excluir ojos a los cuales no se les realizó la tomografía Sirius ${ }^{\mathbb{B}}$ postoperatoria. Por ello, el tamaño de la muestra es relativamente pequeño (92 ojos), lo que hizo que, al dividirlos, quedaran subgrupos con tamaños de muestra de entre 10 y 39 ojos. Sin embargo, para los subgrupos de miopes altos (14 ojos), miopes bajos (39 ojos) e hipermétropes ( 29 ojos), la estimación del poder de las comparaciones pre- y postoperatorias fue del $100 \%$. Para el grupo de ojos con astigmatismo mixto (10 ojos), por otra parte, sí fue solo del 10\%. 


\section{Conclusiones}

El poder corneal obtenido mediante el Mean Pupil Power del tomógrafo Sirius ${ }^{\circledR}$ (MPP), promediado con los valores postoperatorios de la queratometría manual $\left(\mathrm{Km}_{\mathrm{pop}}\right)$ o del SimK Sirius $^{\circledR}$, puede ser muy similar al valor obtenido a partir de la queratometría derivada por el método de la historia clínica $\left(\mathrm{K}_{\mathrm{DHC}}\right)$, en pacientes que han sido sometidos a cirugía fotorrefractiva con láser excimer para miopía. Es necesario realizar estudios adicionales de intercambiabilidad entre las pruebas que permitan establecer, en el futuro, con un mayor número de ojos, su aplicación a casos que requieran cirugía de facoemulsificación más implante de lente intraocular.

\section{Consideraciones éticas}

Protección de personas y animales. Los autores declaran que en esta investigación no se han realizado experimentos en seres humanos ni en animales.

Confidencialidad de los datos. Los autores declaran que han seguido los protocolos de su centro de trabajo sobre publicación de datos de pacientes.

Derecho a la privacidad y consentimiento informado. Los autores declaran que en este artículo no aparecen datos de pacientes.

\section{Conflicto de intereses}

Los autores declaran no detener conflicto de interés.

\section{Referencias}

1. Holladay JT. Cataract surgery in patients with previous keratorefractive surgery (RK, PRK, and LASIK). Ophthalmic Practice. 1997;15:238-44 p.

2. Haigis W. Intraocular lens calculation after refractive surgery. European Ophthalmic Review. 2012;6(1):21-4. doi: 10.17925/EOR.2012.06.01.21

3. Kalyani SD, Kim A, Ladas JG. Intraocular lens power calculation after corneal refractive surgery. Curr Opin Ophthalmol. 2008;19(4):357-62. doi: 10.1097/ICU.0b013e3282fec43e

4. Holladay JT, Galvis V, Tello A. Re: Wang et al.: Comparison of newer intraocular lens power calculation methods for eyes after corneal refractive surgery (Ophthalmology 2015;122:2443-9).
Ophthalmology. 2016;123(9):55-6. doi: 10.1016/j. ophtha.2016.02.038

5. Holladay JT. Consultations in refractive surgery: IOL calculations following radial keratotomy surgery. Refract Corneal Surg. 1989;5: p. 203.

6. Savini G, Calossi A, Camellin M, Carones F, Fantozzi M, Hoffer KJ. Corneal ray tracing versus simulated keratometry for estimating corneal power changes after excimer laser surgery. J Cataract Refract Surg. 2014;40(7):1109-15. doi: 10.1016/j. jers.2013.11.032

7. Holladay JT, Hill WE, Steinmueller A. Corneal Power Measurements Using Scheimpflug Imaging in Eyes With Prior Corneal Refractive Surgery. J Refract Surg. 2009;25(10):862-8. doi: 10.3928/1081597X20090917-07

8. Sónego S, López G, Beaujon OV, Arce CG, Schor P, Campos M. A direct method to measure the power of the central cornea after myopic laser in situ keratomileusis. Arch Ophthalmol. 2004;122(2):15966. doi: 10.1001/archopht.122.2.159

9. Gelender H. Orbscan II-assisted intraocular lens power calculation for cataract surgery following myopic laser in situ keratomileusis (an American Ophthalmological Society thesis). Trans Am Ophthalmol Soc. 2006;104:402-13.

10. Brooks CW. High-Powered Lenses. In: Understanding Lens Surfacing. ButterworthHeinemann. Stoneham, United States. 1992. p. 241245. doi: 10.1016/B978-0-7506-9177-2.50019-9

11. Altman DG, Bland JM. Measurement in medicine: the analysis of method comparison studies. Journal of the Royal Statistical Society. 1983;32(3):307-17. doi: $10.2307 / 2987937$

12. Bland JM, Altman DG. Statistical methods for assessing agreement between two methods of clinical measurement. Lancet. 1986;327(8476):30710. doi: 10.1016/S0140-6736(86)90837-8

13. Bland JM, Altman DG. Measuring agreement in method comparison studies. Stat Methods Med Res. 1999; 8(2):135-60. doi: $10.1177 / 096228029900800204$

14. Giavarina D. Understanding Bland Altman analysis. Biochem Med. 2015;25(2):141-151. doi: 10.11613/BM.2015.015

15. Galvis V, Tello A, Jaramillo LC, Castillo AM, Pareja LA, Camacho PA. Cambios corneales producidos por la cirugía refractiva con excimer láser: revisión de tema. MÉD. UIS. 2017;30(1):99-105. doi: 10.18273/revmed.v30n1-2017010 